(RESEARCH ARTICLE)

\title{
Some data of wavelet analysis on the overall dynamics of the dead and recovered during infection COVID-19
}

\author{
Vyacheslav Lyashenko 1, Eltayib Siddig Mohammed Saad ${ }^{2}$ and Asaad Mohammed Ahmed Babker 3,* \\ ${ }^{1}$ Department of Informatics, Kharkiv National University of RadioElectronics, Ukraine. \\ ${ }^{2}$ Future Lab Medical Laboratories Co. Ltd., Al Kharj, KSA. \\ ${ }^{3}$ Department of Hematology, College of Medical Laboratory Sciences, University of Science and Technology Umdurman, \\ Sudan.
}

Publication history: Received on 22 April 2020; revised on 04 May 2020; accepted on 06 May 2020

Article DOI: https://doi.org/10.30574/wjarr.2020.6.2.0119

\begin{abstract}
Coronaviruses are one of the dangerous sources of human infection. Such a source can be fatal. At the same time, there are certain difficulties in the treatment of diseases that are associated with coronavirus. To solve such issues, it is important to obtain reliable statistics. Equally important is the analysis of the data that are available. Moreover, the necessary condition for the use of such data is the ability to explain the existing relationships between the various parameters that describe the dynamics of the pandemic. The disclosure of these issues is the subject of this study.
\end{abstract}

Keywords: Viruses; COVID-19; Wavelet analysis; Wavelet coherence; Statistics

\section{Introduction}

Viruses are a non-cellular infectious agent that is a constant companion of human life [1]. At the same time, viruses are the most numerous biological forms [2]. Viruses infect all types of organisms and can cause various infectious diseases [3].

The most dangerous form of viruses is coronaviruses. Coronaviruses is a special family of viruses, which includes about 40 types of RNA-containing viruses $[4,5]$. Coronaviruses have a high pathogenicity, the ability to overcome human immunity. For example, COVID-19 showed the ability to spread rapidly. At the same time, the ability to mutate complicates the ability to quickly develop tools to combat coronaviruses. All this necessitates the conduct of various studies to counter coronaviruses. Among such studies, a statistical analysis of data on the course of diseases obtained from exposure to coronavirus can be distinguished. Such an analysis allows us to study the processes of the course of diseases, study the dynamics of diseases, and reveal various relationships. These questions are the subject of this work.

\section{Material and methods}

\subsection{A small review of research publications}

COVID-19 has been the subject of many publications [6-14]. Static analysis of the COVID-19 pandemic occupies a special place in such studies. For this, classical methods are used, as a rule. This allows you to quickly assess the situation and give some recommendations.

In [6], based on stochastic modeling, the dynamics of COVID-19 propagation in China was considered. The analysis is based on open data and allows you to simulate the development of the epidemic.

\footnotetext{
* Corresponding author: Asaad Mohammed Ahmed Babker
} 
H. Nishiura, N. M. Linton, and A. R. Akhmetzhanov examine the reliability of empirical data [7]. To do this, statistical methods are used to compare complete data sets and individual subsets of the source data. Such a comparison is based on the construction of models for analyzing the distribution of empirical data. This minimizes errors in predicting the development of the epidemic.

In [8], various distributions of primary data are also used to simulate the possible trajectories of the epidemic. The distribution model is determined by daily data. This allows you to build an accurate forecast.

The choice of data distribution methods that describe the dynamics of the COVID-19 epidemic are considered in studies $[9,10]$. The selection of such methods is based on accurate statistical criteria and estimates between different groups of data.

D. Baud, X. Qi, K. Nielsen-Saine, D. Muss, L. Poma and G. Favre consider simple descriptive statistics to analyze COVID19 epidemic data [11]. At the same time, various graphs are used to visualize such data. A similar approach is considered in the study [12]. This allows us to present the process of the development of the epidemic as a whole.

Studies should also be highlighted that address the relationship of different markers in the study of the development of the COVID-19 epidemic. This allows us to evaluate the influence of individual factors on the development of COVID-19, as well as to examine the relationship between various parameters of concomitant diseases. For this, various data visualization methods are used, as well as a correlation analysis method to identify possible relationships between individual COVID-19 epidemic markers. An example of such studies is the work $[13,14]$.

Nevertheless, the development of the COVID-19 pandemic makes it necessary to use various methods that allow for appropriate analysis. Moreover, among such methods, it is worth highlighting those that allow mutual analysis of various indicators. Among these methods, wavelet analysis can be distinguished [15, 16, 17]. This analysis allows us to identify implicit trends, to analyze the mutual influence of various indicators in the development of the COVID-19 pandemic.

\subsection{Wavelet coherency as a data analysis tool}

Among the methods of wavelet analysis, we should single out a method that is based on the estimation of wavelet coherence [15-18]. This method allows you to do cross-analysis for the data series that are being investigated. The basis of this analysis is the search for points of sharp transition from one value to another value.

To determine the values of wavelet coherence, we consider the values of cross wavelet spectra $W_{x y}(g, q)$ for two data series $g(t)$ and $q(t)$ ( $x$ is the variable that displays the data number in the series under investigation and $y$ is the variable that displays the depth of cross-links for a time series $-t$ ). The general formula has the following form [19, 20]:

$$
R^{2}(g, q)=\frac{\left|V\left(k^{-l} W_{x y}(g, q)\right)\right|}{V\left(k^{-1}\left|W_{x}(g, q)\right|^{2}\right) V\left(k^{-1}\left|W_{y}(g, q)\right|^{2}\right)},
$$

where: $V$ is a smoothing operator, $0 \leq R^{2}(g, q) \leq 1$.

To calculate the values of the wavelet coherence, we use the Morlet wavelet. If these values tend to zero, then we have a weak correlation. Otherwise, we have a strong correlation $[18,19]$.

\subsection{Data for analysis}

For an appropriate analysis, we consider the general dynamics of the number of diseased people who died and recovered from infection with COVID-19. We will consider these data in the period 18.01.2020-01.04.2020 (generalized data from the site https://www.who.int/emergencies/diseases/novel-coronavirus-2019). In addition, we note that we are considering the following data type: data that reflect the cumulative total (Figure 1), data in the context of each day from the studied interval (Figure 2). 


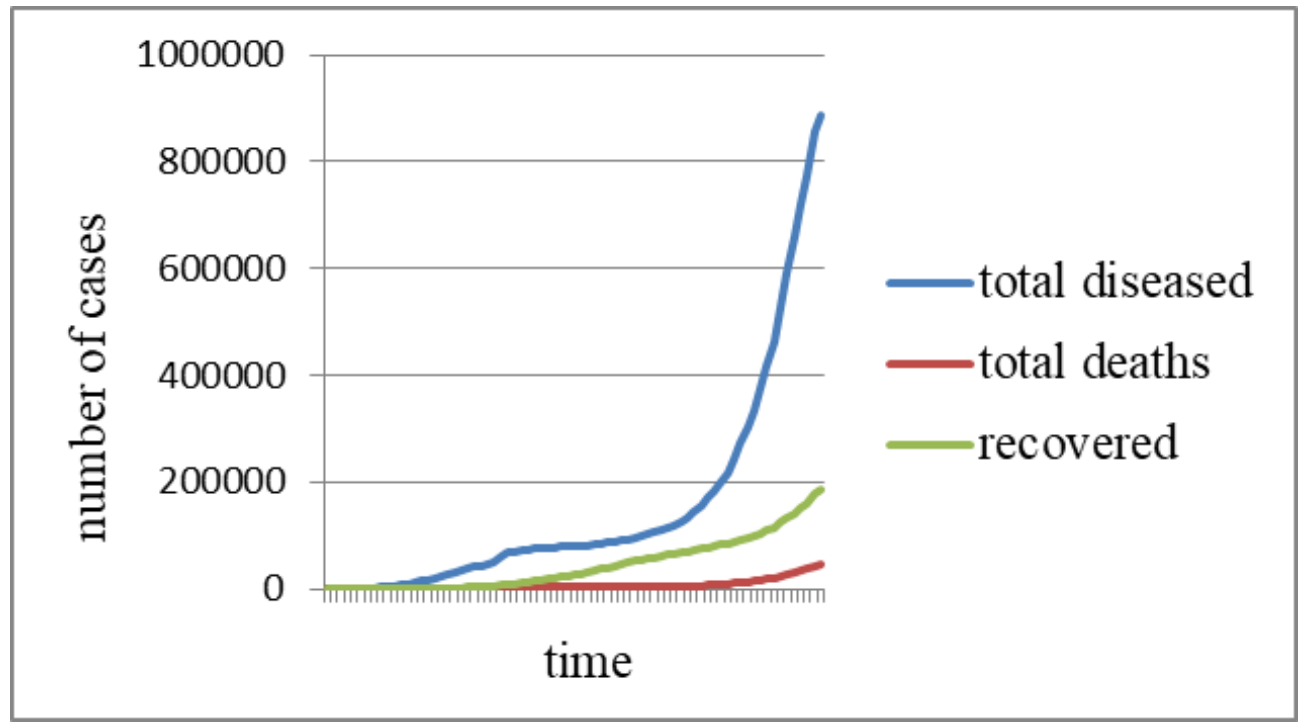

Figure 1 Statistics on COVID-19 (reflect the cumulative total)

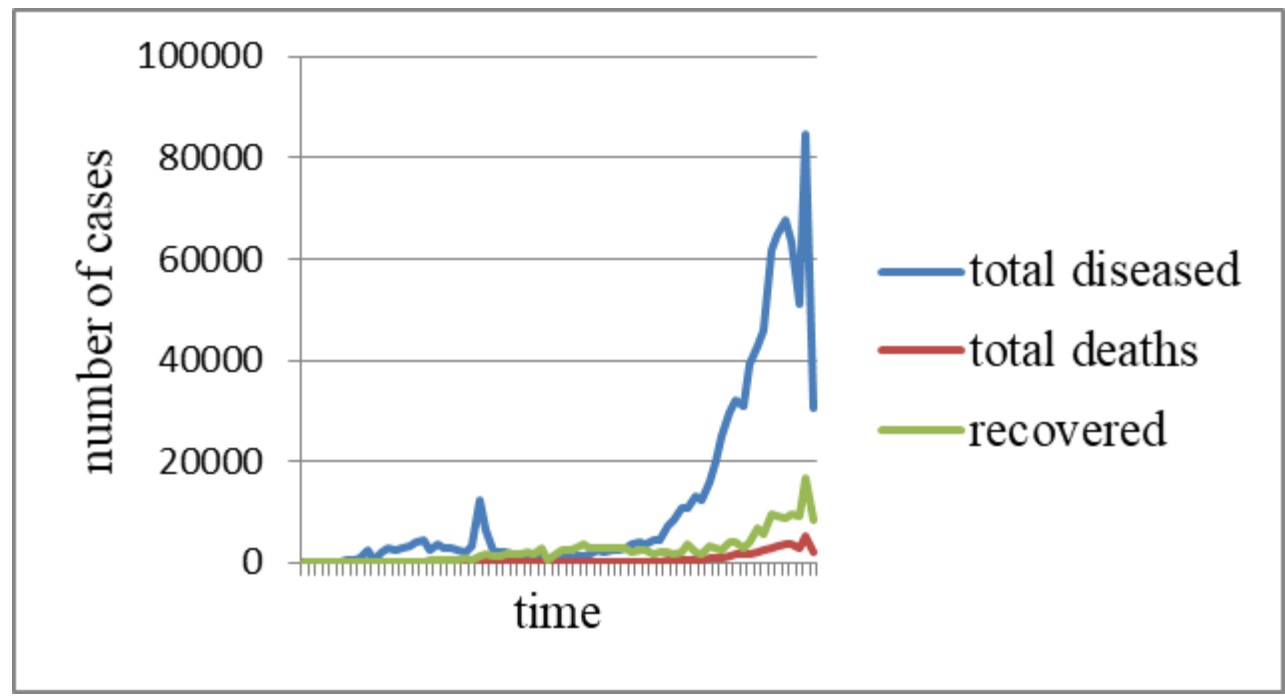

Figure 2 Statistics on COVID-19 (data in the context of each day from the studied interval

We see some differences in the data of Figure 1 and Figure 2. Next, we consider these data through estimates of the values of wavelet coherence.

\section{Results and discussion}

Figure 3 - Figure 6 shows the results of wavelet coherence estimates. The dashed white line limits the region of reliable values of wavelet coherence (with a confidence level of at least 0.95). Figure 3 - Figure 6 also shows a scale for analyzing the significance of wavelet coherence data. Such data for clarity also have color values.

Figure 3 shows the wavelet coherence between the total number of COVID-19 cases and the number of deaths from COVID-19 (these values are shown for the data in Figure 1, reflect the cumulative total). 


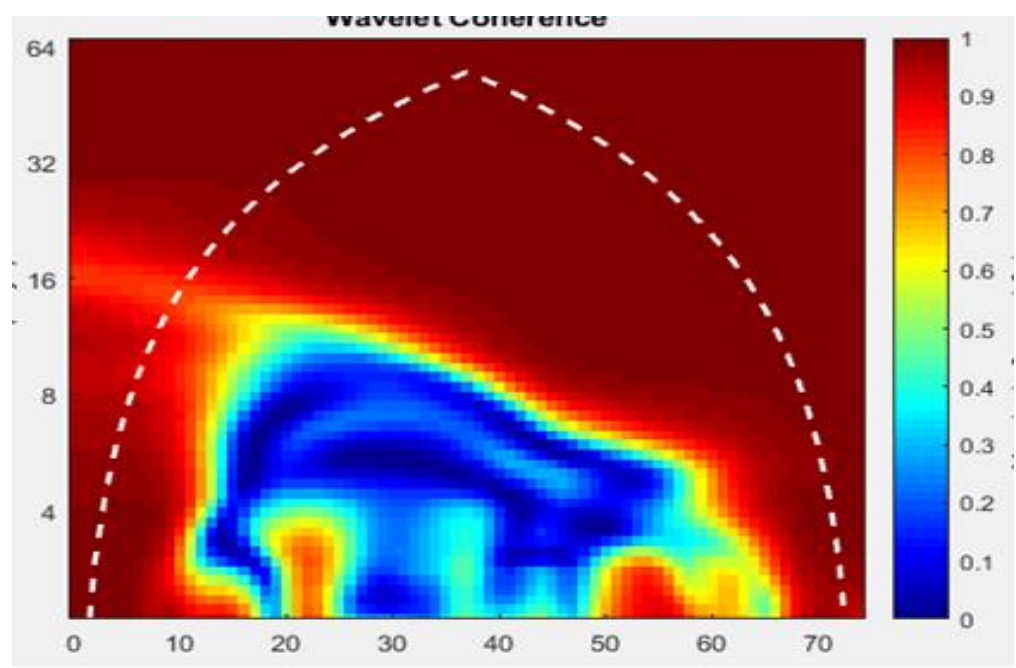

Figure 3 Wavelet coherence between the total number of patients with COVID-19 and the number deaths from COVID-19 (for data in Figure 1)

Figure 4 shows the wavelet coherence between the total number of cases of COVID-19 and the number of recovered from COVID-19 (these values are shown for the data in Figure 1, reflect the cumulative total).

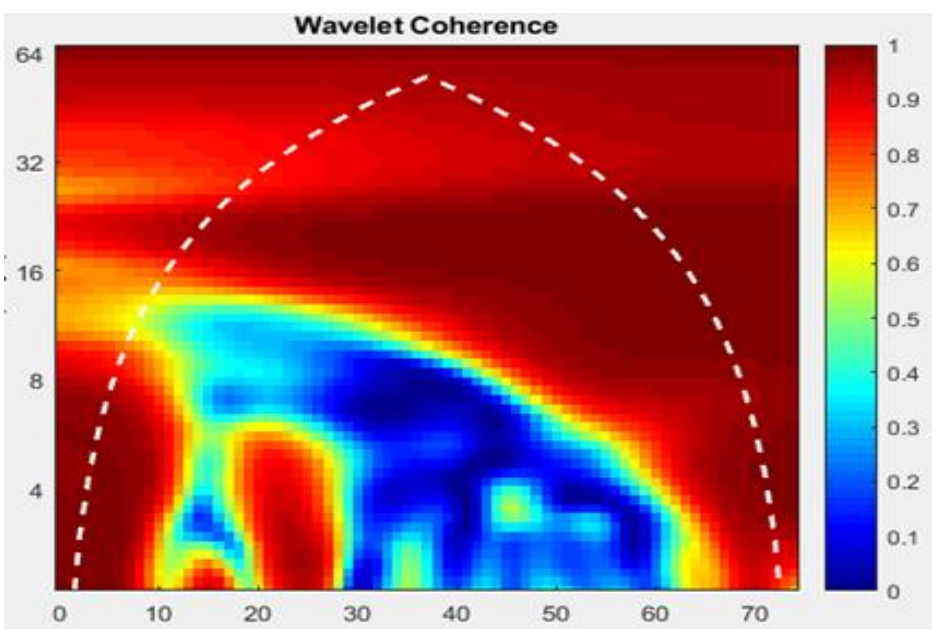

Figure 4 Wavelet coherence between the total number of patients with COVID-19 and the number of patients recovered from COVID-19 (for the data in Figure 1)

First of all, it should be noted that the data in Figure 3 and Figure 4 are identical. At the beginning of the development of the COVID-19 pandemic, the wavelet coherence between the data under study is significant across almost the entire cross-reference depth. Then, the significance of wavelet coherence in the depth of cross-references begins from the 15 th day of observation. Therefore, we can conclude:

at the beginning of the development of the COVID-19 pandemic, not all data were displayed in their statistics,

deaths and recovered patients occur at least 15 days after the course of COVID-19 disease.

In recent periods, an increase in the significance of wavelet coherence over almost the entire depth of cross-references has also been observed (see Figure 3 and Figure 4). The explanation for this fact should indicate the continuation of the COVID-19 pandemic. Therefore, the data at the end of the study period may give some error.

Figure 5 shows the wavelet coherence between the total number of COVID-19 cases and the number of deaths from COVID-19 (these values are shown for the data in Figure 2, data in the context of each day from the studied interval). 


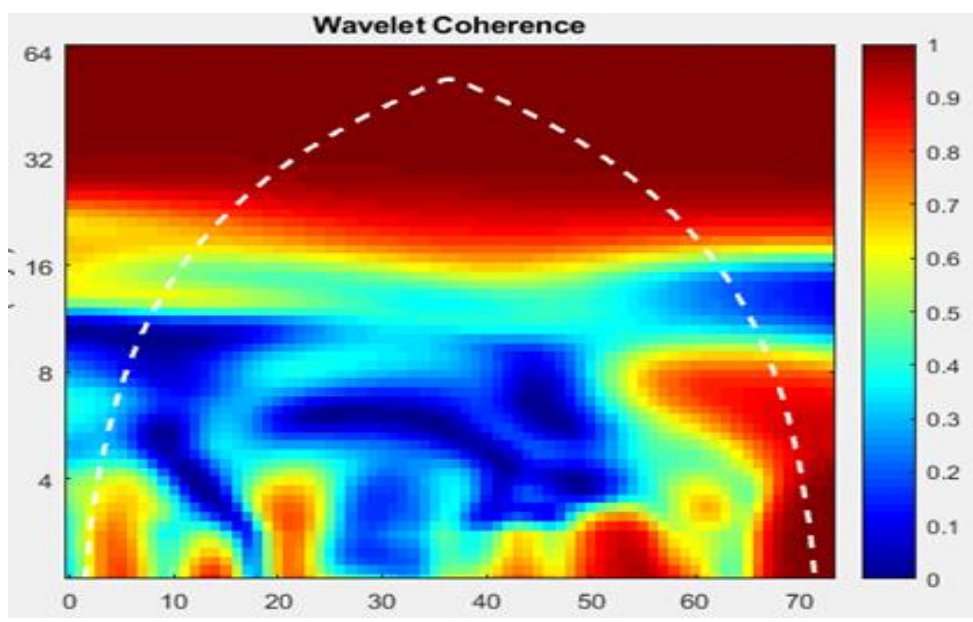

Figure 5 Wavelet coherence between the total number of patients with COVID-19 and the number deaths from COVID-19 (for data in Figure 2)

Figure 6 shows the wavelet coherence between the total number of cases of COVID-19 and the number of recovered from COVID-19 (these values are shown for the data in Figure 2, data in the context of each day from the studied interval).

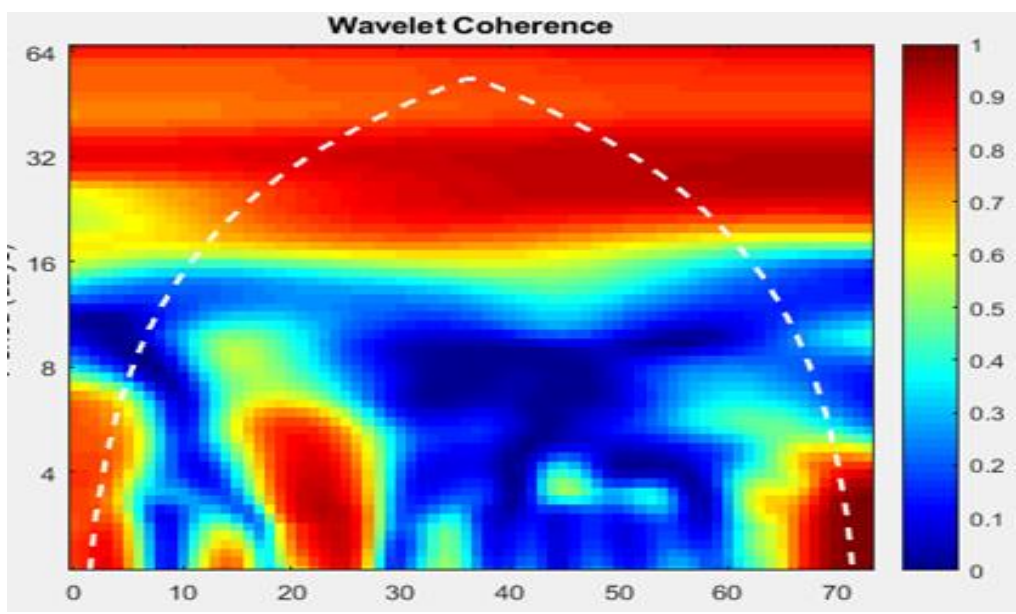

Figure 6 Wavelet coherence between the total number of patients with COVID-19 and the number of patients recovered from COVID-19 (for the data in Figure 2)

Analysis of the data in Figure 5 and Figure 6 also allows us to draw some conclusions:

Presence fragmentary portions, for which is characteristic of minor time frames. For these areas the depth crossreference in the range to 4 (8) days. This can be attributed either to the rapid onset of death (in view of possible complications) or to a quick recovery (in view of the late confirmation of COVID-19), According to the depth of crossreferences, starting from the 16th day of observations, the values of wavelet coherence are significant for the entire interval that is studied. Therefore, the previous conclusion that deaths and patients recover at least 15 days after the course of COVID-19 disease is true.

\section{Conclusion}

We examined the possibility of using wavelet coherence to study the dynamics of the COVID-19 pandemic. As data, we consider the dynamics of the number of sick people who died and recovered from infection with COVID-19. This data is considered as data that reflect the cumulative total and as data in the context of each day from the studied interval. We obtained results that explain some points in the dynamics of the COVID-19 pandemic. In particular, it was found that not all data are statistically complete. It is also shown that the course of the disease COVID-19 has its results (death or recovery) on average after 15-16 days. 


\section{Compliance with ethical standards}

\section{Disclosure of conflict of interest}

Disclosure of conflict of interest.

\section{References}

[1] De Farias ST, Jheeta S and Prosdocimi F. (2019). Viruses as a survival strategy in the armory of life. History and philosophy of the life sciences, 41(4), 41-45.

[2] Nefedova LN and Kim AI. (2018). Evolution of the Mechanisms of Virus-Cell Interactions. Biology Bulletin Reviews, 8(4), 292-299.

[3] Koonin EV. (2016). Viruses and mobile elements as drivers of evolutionary transitions. Philosophical Transactions of the Royal Society B: Biological Sciences, 371(1701), 20150442.

[4] Klinov D, Isaeva E, Koroleva L, Morozova O and Silnikov V. (2016). Inhibition of RNA-containing viruses by artificial RNases. Drug Target Review, (2), 49-54.

[5] Saif YM, Guy JS, Day JM, Cattoli G and Hayhow CS. (2020). Viral enteric infections. Diseases of Poultry, 401-445.

[6] Peng L, Yang W, Zhang D, Zhuge C and Hong L. (2020). Epidemic analysis of COVID-19 in China by dynamical modeling. arXiv preprint arXiv, 06563.

[7] Nishiura H, Linton NM and Akhmetzhanov AR. (2020). Serial interval of novel coronavirus (COVID-19) infections. International journal of infectious diseases, 93, 284-286.

[8] Zhang S, Diao M, Yu W, Pei L, Lin Z and Chen D. (2020). Estimation of the reproductive number of Novel Coronavirus (COVID-19) and the probable outbreak size on the Diamond Princess cruise ship: A data-driven analysis. International journal of infectious diseases, 93, 201-204.

[9] Shi H and et. al. (2020). Radiological findings from 81 patients with COVID-19 pneumonia in Wuhan, China: a descriptive study. The Lancet Infectious Diseases, 20, 425-434.

[10] Jung SM and et. al. (2020). Real-time estimation of the risk of death from novel coronavirus (COVID-19) infection: Inference using exported cases. Journal of clinical medicine, 9(2), 523.

[11] Baud D, Qi X, Nielsen-Saines K, Musso D, Pomar L and Favre G. (2020). Real estimates of mortality following COVID-19 infection. The Lancet Infectious Diseases.

[12] Remuzzi A and Remuzzi G. (2020). COVID-19 and Italy: what next? The Lancet.

[13] Fang Y, Zhang H, Xie J, Lin M, Ying L, Pang P and Ji W. (2020). Sensitivity of chest CT for COVID-19: comparison to RT-PCR. Radiology, 200432.

[14] Ai T and et al. (2020). Correlation of chest CT and RT-PCR testing in coronavirus disease 2019 (COVID-19) in China: a report of 1014 cases. Radiology, 200642.

[15] Abd Elgadir A, Babker AM, Osman AL, Ismail M and Lyashenko V. (2019). New Approach for Analysis the Correlation of Some Oxidative Markers in Type 2 Diabetes Mellitus by Data Wavelet Analysis. Indian Journal of Public Health, 10(11), 2449-2455.

[16] Lyashenko VV, Babker AMAA and Kobylin OA. (2016). Using the Methodology of Wavelet Analysis for Processing Images of Cytology Preparations. National Journal of Medical Research, 6, 98-102.

[17] Ali WM \& et al. (2019). Wavelet coherence as a tool for visualizing the relationship between glomerular filtration rate and renal artery blood flow velocity. International Journal of Emerging Trends in Engineering Research, $7(12), 818-823$.

[18] Lyashenko V, Zeleniy O, Mustafa SK and Ahmad MA. (2019). An Advanced Methodology for Visualization of Changes in the Properties of a Dye. International Journal of Engineering and Advanced Technology, 9(1), 7117114.

[19] Torrence C and Webster PJ. (1999). Interdecadal changes in the ENSO-monsoon system. Journal of climate, 12(8), 2679-2690. 
[20] Ng EK and Chan JC. (2012). Geophysical applications of partial wavelet coherence and multiple wavelet coherence. Journal of Atmospheric and Oceanic Technology, 29(12), 1845-1853.

\section{How to cite this article}

Vyacheslav L, Eltayib Siddig MS and Asaad MB. (2020). Some data of wavelet analysis on the overall dynamics of the dead and recovered during infection COVID-19. World Journal of Advanced Research and Reviews, 6(2), 17-23. 